\title{
Induced Rule-Based Fuzzy Inference System from Support Vector Machine Classifier for Anomalous Propagation Echo Detection
}

\author{
Hansoo Lee, Yeongsang Jeong, and Sungshin Kim
}

\begin{abstract}
Support vector machine is a popular classifier to find an optimal hyperplane which separates given data into two groups. Due to its remarkable performance, the support vector machine is applied in various fields such as inductive inferences, classifications or regressions. By its black box characteristics, there are plenty of actively discussed researches about analyzing trained support vector machine classifier. In this paper, we propose a method to make a fuzzy inference system using extracted rules from the support vector machine. As an object of classification, an anomalous propagation echo is selected which occurs frequently in radar data and becomes the problem in a precipitation estimation process. After applying a clustering method, learning dataset is generated from clusters. Using the learning dataset, a support vector machine is implemented. After that, a decision tree is generated. And it is used to implement fuzzy inference system by rule extraction and input selection. Finally, we can verify and compare performances. With actual occurrence cased of the anomalous propagation echo, the performances of both classification methods showed similar results. Further, we can determine the inner structure of the support vector machine.
\end{abstract}

Index Terms - Support vector machine, rule extraction, fuzzy inference system, anomalous propagation echo.

\section{INTRODUCTION}

Ground-based weather radar is a well-known remote sensing device which is used to observe meteorological phenomena for weather forecasting [1]. The ground-based radar has many advantages. First, it can observe precisely over wide areas with high resolution. Second, it can offer almost real time scanning data. Third, it can provide meaningful and valuable observation results such as reflectivity, Doppler velocity, and so on. However, the weather radar occasionally derives false scan results that do not originate from precipitation. Therefore, it is essential to separate meaningful scanning information from radar data. Fortunately, there is already implemented process in actual environment called a quality control process [2]. However, currently used quality control process tends to depend on the

Manuscript received December 5, 2015; revised February 8, 2016. This research was supported by Basic Science Research Program through the National Research Foundation of Korea (NRF) funded by the Ministry of Education (NRF-2014R1A1A2056958) and was supported by Global Ph. D Fellowship Program through the National Research Foundation of Korea (NRF) funded by the Ministry of Education (2013-034596) and was supported by BK21PLUS, Creative Human Resource Development Program for IT Convergence.

Hansoo Lee, Yeongsang Jeong, and Sungshin Kim are with the Pusan National University, Busan, Republic of Korea (e-mail: \{hansoo, dalpangi03, sskim\} @ pusan.ac.kr). experts' opinion. In other words, the current process has to put up with some drawbacks such as biased and inconsistent decision process.

For solving these problems, there are a lot of ongoing researches about the automated quality control process for supporting the experts' decision making process [2]. The researches focus on the quality control process to separate precipitation and non-precipitation echoes in the radar data The precipitation echoes consist of the observation results related to meteorological phenomena such as rain, snow, clouds, and so on. On the other hands, the non-precipitation echoes consist of non-meteorological materials such as small particles in the atmosphere, interference noises, a false observation caused by refracted beam, and so on. Especially, an anomalous propagation echo [1], [3], occurred by a refracted radar beam, is one of representative non-precipitation echo which have similar properties of precipitation, occur frequently in weather radar observation process and becomes the problems in precipitation estimation processes.

In order to separate the anomalous propagation echo, it is important to choose an appropriate classifier. A support vector machine (SVM) can be considered as one of reasonable choices. The SVM has been proven its remarkable generalization performance in a variety of applications such as classification, regression, and so on. However, the SVM has an inability to provide a detail explanation for the solutions because it is one of the 'black-box' model like artificial neural networks (ANN). Hence, in order to explain the black-box models including SVM and ANN, there have been a lot of researches to extract rules [4].

In this paper, we propose a method to make a fuzzy inference system not only separating the anomalous propagation echo but also describing the SVM classifier. The crisp rules are extracted by a decision tree, and they are converted to fuzzy rules and applied to implement a fuzzy inference system.

The rest of the paper is organized as follows. In Section II, we explain radar data structure and the anomalous propagation echo. And in Section III, we elucidate the entire proposed system which consists of SVM, rule extraction, and fuzzy inference system. After that, the experimental results are described in Section IV. Finally, the conclusion and future work are showed in Section V.

\section{ANOMAlous Propagation Echo}

The observation efficiency of the weather radar is different 
by properties of remote sensing device using radio signal. In other words, a beam path of the weather radar can be changed by the atmospheric condition. It is classified as sub-refraction, normal refraction, super-refraction and ducting according to its refracted path [3]. Among them, when the radar beam is deviated toward the surface by super-refraction or ducting, the resultant echo represents reflection of the ground or the sea surface which is not a meteorological target. It is called as an anomalous propagation echo.

The weather radar computes altitude of observation targets consider as the normal refraction of the radar beam. Therefore, unexpected echoes could appear in the observation region of the weather radar by a surface scattering when the super-refraction or the ducting occurs. The surface scattering makes the weather radar to consider trees, mountains, buildings and sea surface as meteorological objects such as rain, snow, etc.

It is one of the representative contamination source in the forecasting process because it induces a severe problem in quantitative precipitation estimation. The anomalous propagation echo should be removed from radar data because the echo originating from the surfaces can be misinterpreted as heavy precipitation. In short, the refracted signals may lead to large overestimates of precipitation totals by the radar beam seeing Earth's surface instead of the atmosphere. Also, its location is difficult to predict. Furthermore, when the radar beam refracts toward the surface more severely, the intensity and extension of clutter areas can also change [1].

\section{PROPOSED ClASSIFICATION METHOD}

\section{A. Overall Process for Anomalous Propagation Echo Classification}

The entire proposed system is shown in Fig. 1. The detailed sequence is described below. First of all, we need to clarify why we select a corrected reflectivity (CZ) and a Doppler velocity data (VR). There are several kinds of useful information in raw radar data. According to recent research for the anomalous propagation echo classification, the echo has following properties [5]: a near-zero radial velocity, a low spectrum width, a high texture of the reflectivity field, and so on. In this reason, we select the corrected reflectivity and a Doppler velocity data as input features.

Due to observation principles of the weather radar, the raw radar data follow the spherical coordinate. Therefore, in order to analyze the radar data intuitively, a coordinate conversion process is done from spherical to Cartesian. This process makes to apply a clustering algorithm for grouping individual point data. Also, in order to find and extract same coordinates, the coordinate conversion process is done to both the corrected reflectivity and Doppler velocity data.

After the coordinate conversion, a spatial clustering method [6] which is one of hierarchical clustering methods is applied for grouping the reflectivity data. The clustered data are easier to deal with than the raw data because the radar data include millions of data points. The proposed system uses statistical features derived from the clusters such as mean, minimum, maximum and its centroid position.

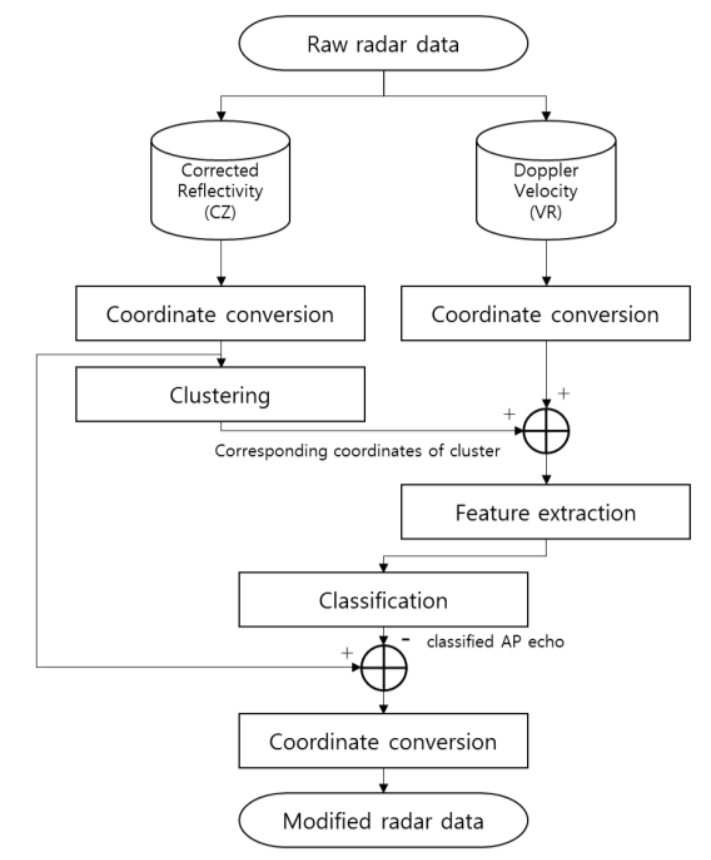

Fig. 1. Overall process for anomalous propagation echo separation.

In feature extraction process, five properties are derived and used as inputs: centroid altitude of the cluster $\left(x_{1}\right)$, average reflectivity $\left(x_{2}\right)$, maximum reflectivity $\left(x_{3}\right)$, average Doppler velocity $\left(x_{4}\right)$, and minimum Doppler velocity $\left(x_{5}\right)$. The reason why we select the centroid altitude of the cluster is that the anomalous propagation echo appears in low altitude by its own properties.

When the feature extraction process is done, the proposed method is applied as binary classification method. The detailed sequences are described in next section. Using the classifiers, each cluster is classified +1 or -1 , which indicates that the selected cluster is anomalous propagation echo or not, respectively. The classified clusters which determined as an anomalous propagation echo are removed from the corrected reflectivity.

After the removal process is done, the reverse coordinate conversion process is applied: from Cartesian to spherical coordinate. Finally, the processed radar data without the anomalous propagation echo is generated.

\section{B. Support Vector Machine}

A SVM is a binary classification method that divides the given data into two groups in the best possible way by using hyperplanes. This method is based on a structural risk minimization method to reduce the error rather than the empirical risk minimization method used in traditional statistical learning theory. In other words, after the division of an entire group into subgroups, a decision function is selected. This function can minimize the empirical risk for the subgroups. Thus, the SVM method has the advantage of achieving great performance in classification, prediction, and estimation processes by using a relatively low amount of the given learning data [7]-[9].

The SVM classifier aims to find a hyperplane which has maximum margin between two classes. As shown in (1), a quadratic programming problem can derive the optimized hyperplane where $\omega$ is weight vector, $b$ is bias, and $y_{i}$ is a class of $x_{i}$. 


$$
\begin{aligned}
& \left.\operatorname{minimize}_{<\omega \cdot x+b>}<\omega \cdot \omega\right\rangle \\
& \text { subject to } y_{i}\left(<\omega \cdot x_{i}>+b\right) \geq 1
\end{aligned}
$$

It can be rewritten as (2) using Lagrange multiplier $\alpha$. And as shown in (3) and (4), the optimized weight vector and bias can be obtained.

$$
\begin{gathered}
\underset{\substack{i=1, \cdots, n \\
\text { maximize }}}{\mathrm{W}(\alpha)=} \sum_{i=1}^{n} \alpha_{i}-\frac{1}{2} \sum_{i=1}^{n} \sum_{j=1}^{n} y_{i} y_{j} \alpha_{i} \alpha_{j}<x_{i} \cdot x_{j}> \\
\omega^{*}=\sum_{i=1}^{n} \alpha_{i}^{*} y_{i} x_{i} \\
b^{*}=-\frac{\max _{y_{i}=-1}\left(\left\langle w^{*} \cdot x_{i}\right\rangle\right)+\min _{y_{i}=-1}\left(\left\langle w^{*} \cdot x_{i}\right\rangle\right)}{2}
\end{gathered}
$$

However, if the dataset is not linear, the SVM projects the original dataset into higher dimension using a function $\phi(\cdot)$ as shown in (5). In this case, when a kernel function is known, it is unnecessary to figure out the result of individual projection data in (6). It is called kernel trick.

$$
\begin{aligned}
& \sum_{i=1}^{n} \alpha_{i}^{*} y_{i} \phi\left(x_{i}\right)^{T} \phi\left(x_{j}\right)+\mathrm{b}^{*} \\
& K\left(x_{i}, x_{j}\right)=\phi\left(x_{i}\right)^{T} \phi\left(x_{j}\right)
\end{aligned}
$$

There are several kernel functions such as linear, polynomial, Gaussian radial basis function, and so on. Using these equations and concepts, the SVM classifier has powerful performance.

\section{Induced Rule-Based Fuzzy Inference System}

SVM classifiers are exceedingly powerful tools. However, due to its characteristics that cannot provide appropriate explanations how the classification results are derived, there are lots of researches to elucidate implemented SVM classifiers [3]. There are two of representative analysis researches: sequence-covering approach using support vector [10], and decision tree approach [11], [12]. Especially decision tree is a sort of white-box models, which easily comprehensible and analyze inner structure.

In this paper, we use the decision tree approach to analyze the SVM and establish a fuzzy inference system. The entire sequences of the rule extraction is as follows. First, the learning data are normalized and divided into three pieces: training, verification, and testing. Second, a SVM classifier is generated using the training dataset, represented as dataset $\mathrm{A}$. Third, the verification dataset, which indicates dataset B in Fig. 2, is applied into the generated SVM classifier. Then the verification dataset through the SVM classifier can be started to consider as representing the SVM because the classified result have errors of the SVM. Fourth, using the derived dataset, which indicates artificial dataset B', a decision tree is generated. Fifth, a series of crisp rules is generated from the decision tree. Finally, we can get a fuzzy inference system using the crisp rules. The entire process is described in Fig. 2.
The SVM, decision tree, and fuzzy inference system can be verified using the testing dataset, as known as dataset $\mathrm{C}$.

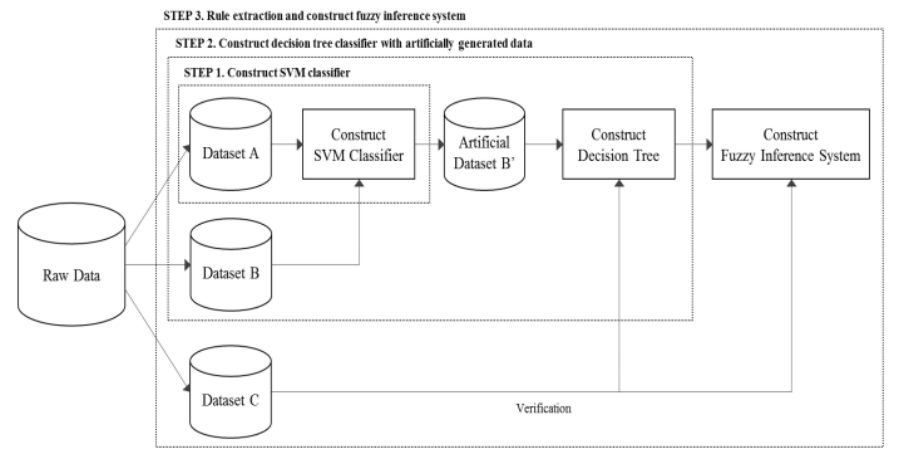

Fig. 2. Entire process to establish fuzzy inference system from support vector machine.

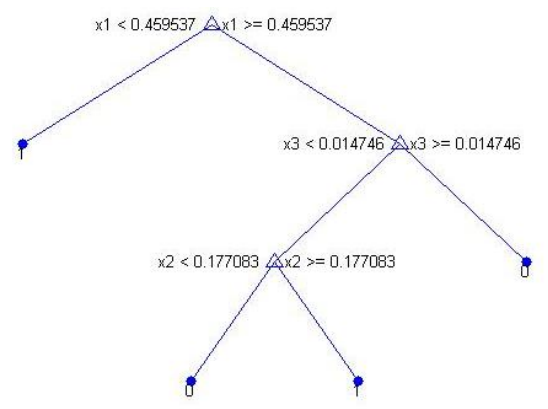

Fig. 3. An actual implementation of decision tree.
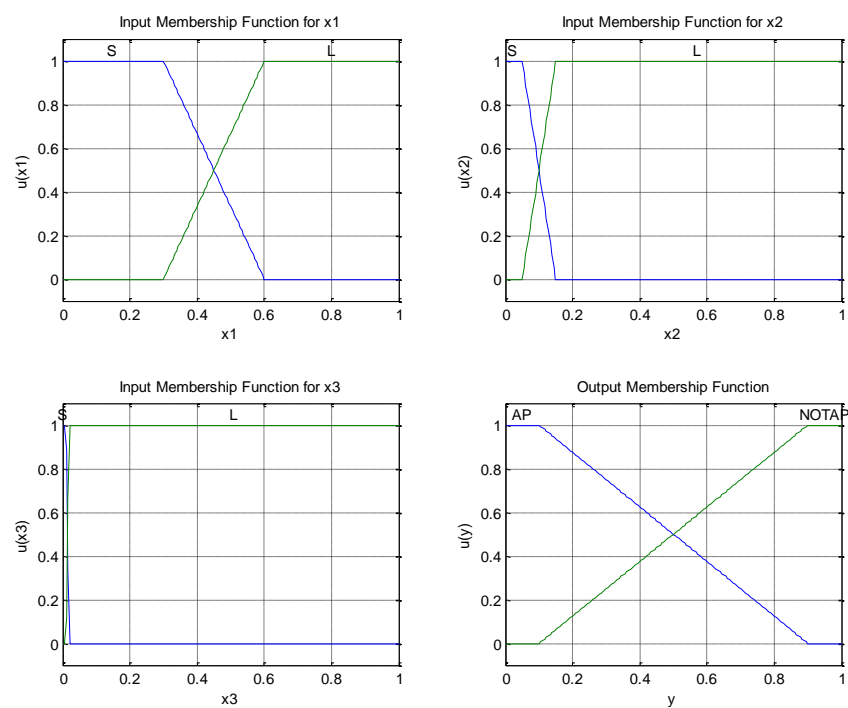

Fig. 4. Input and output membership functions of fuzzy inference system.

Fig. 3 shows an example of decision tree for deriving a fuzzy inference system. As mentioned earlier, we applied 5 properties as inputs of the SVM. And the decision tree indicates there is only 3 important inputs for separating anomalous propagation echo. Using the decision tree, we can construct fuzzy rules as follows.

Rule 1: If $x_{1}$ is small, then $y$ is AP.

Rule 2: If $x_{1}$ is large and $x_{3}$ is large, then $y$ is NOTAP.

Rule 3: If $x_{1}$ is large and $x_{2}$ is small and $x_{3}$ is large, then $y$ is NOTAP.

Rule 4: If $x_{1}$ is large and $x_{2}$ is large and $x_{3}$ is large, then $y$ is AP.

The input and output membership functions are trapezoidal 
shaped function. And each parameter is adjusted using the crisp rules from the decision tree.

\section{EXPERIMENTAL RESULTS}

For the purpose of verifying the proposed system, this paper selected actual appearance cases for training and testing A confusion matrix is applied in this paper for verifying the accuracy as shown in equation (7).

$$
\operatorname{Accuracy}(\%)=\frac{T P+T N}{T P+T N+F P+F N}
$$

In this equation, each parameter indicates as follow: TP for true positive, TN for true negative, FP for false positive, and $\mathrm{FN}$ for false negative.

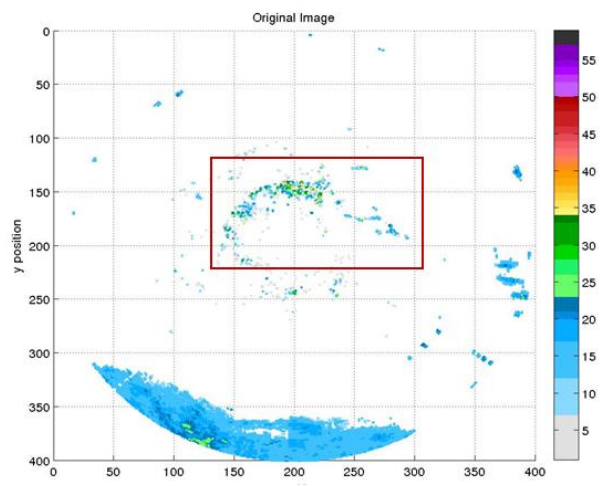

(a)

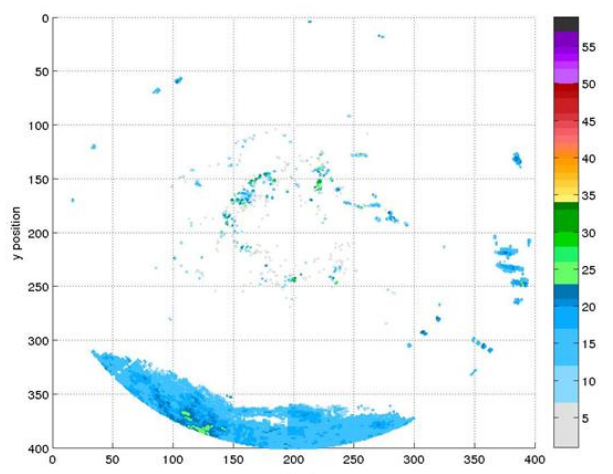

(b)

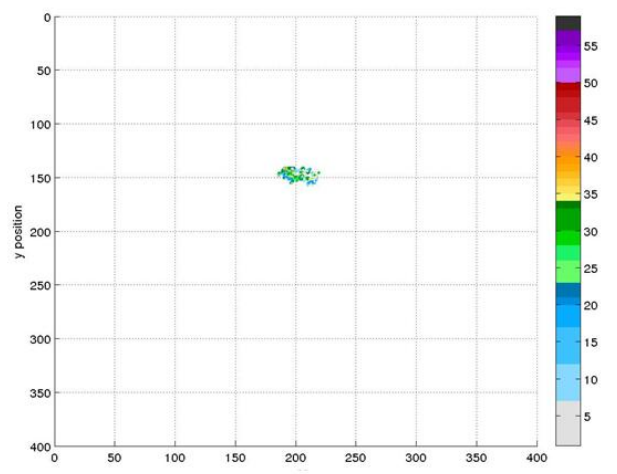

(c)

Fig. 5. Anomalous propagation echo occurrence case 1: (a) original image, (b) image without classified echo, (c) only classified echo image.

The true parameter indicates the anomalous propagation echo. And the false parameter is the other echoes. We select three radar sites for experiments. The average accuracy of the SVM classifier is $91.59 \%$. On the other hands, the average accuracy of the induced rule-based fuzzy inference system is $87.49 \%$. In conclusion, we can consider that the important variables are centroid altitude, average reflectivity, and maximum reflectivity data in this case. Using these variables, we can build similar, although slightly lower performance, classifier for separating the anomalous propagation echo.

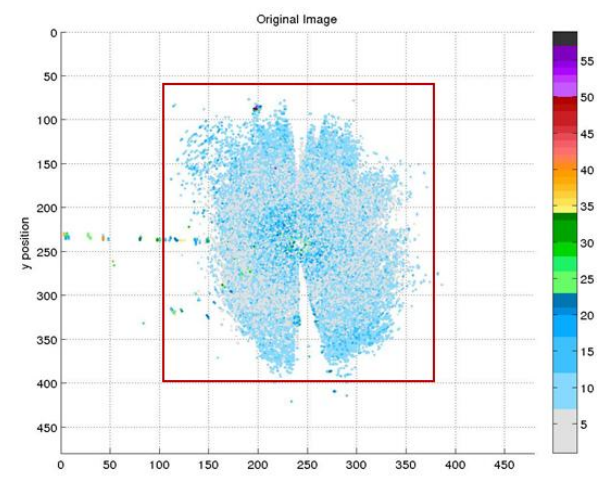

(a)

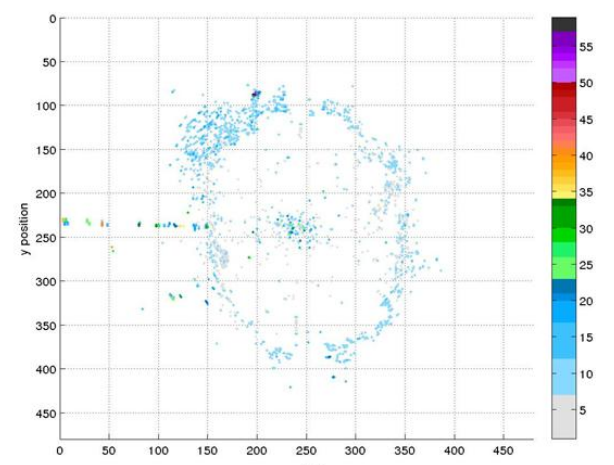

(b)

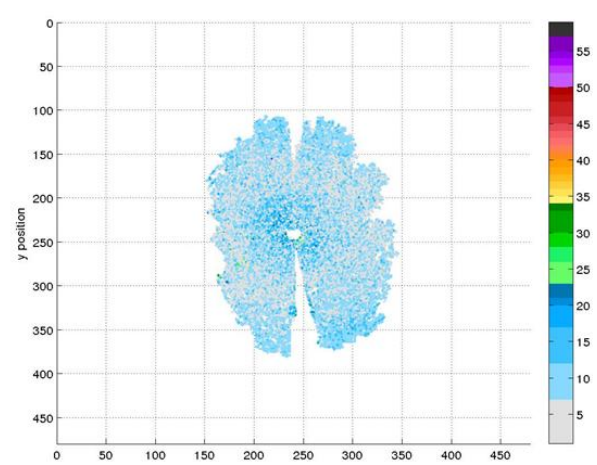

(c)

Fig. 6. Anomalous propagation echo occurrence case 1: (a) original image, (b) image without classified echo, (c) classified echo image.

Fig. 5 and Fig. 6 show the classification results using the implemented fuzzy inference system with actual appearance cases of the anomalous propagation echo. The case shown in Fig. 5 indicates a case with precipitation echo region shown below. As shown in Fig. 5(a), a squared mark on the middle region represents as the anomalous propagation echo. Fig. 5(b) shows the radar image without the classified anomalous propagation echo by the proposed system. And Fig. 5(c) describes the separated anomalous propagation echo.

The case shown in Fig. 6 indicates an independent appearance case of the anomalous propagation echo. As 
shown in Fig. 6(a), the entire region in the squared marks represent as the anomalous propagation echo. Fig. 6(b) shows the radar image without the classified anomalous propagation echo by the proposed method. And Fig. 6(c) shows the anomalous propagation echo only. From the figures, it is confirmed that the most of the regions of the anomalous propagation echo are removed. In conclusion, the induced rule-based fuzzy inference system from the support vector machine can be evaluated well according to these experiment results and accuracy comparison results.

\section{CONCLUSION}

In weather forecasting process, it is important to analyze the radar data accurately. Among the non-precipitation echoes, the anomalous propagation echo is one of the representative non-precipitation echoes. This paper proposes the fuzzy inference system with induced rules from the SVM. The five different properties derived by clustering algorithm are applied as inputs of the classifiers selectively. We can conclude that the fuzzy inference system can detect the anomalous propagation echo with similar accuracy as the SVM.

Further proposed work is to improve accuracy of detecting the anomalous propagation echo. Also, the other classification method could be applied such as artificial neural network, naïve Bayesian classifier, and so forth. The empirical study is needed to select most appropriate algorithm for the anomalous propagation echo. Finally, the proposed system could be applied to other non-precipitation echoes.

\section{REFERENCES}

[1] M. A. Rico-Rameriz and I. D. Cluckie, "Classification of ground clutter and anomalous propagation using dual-polarization weather radar," IEEE Trans. Geosci Remote, vol. 46, no. 7, pp. 1892-1904, July 2008.

[2] V. Lakshmanan, A. Fritz, T. Smith, K. Hondl, and G. Stumpf, "An automated technique to quality control radar reflectivity data," J. Appl Meteorol Clim, vol. 46, no. 3, pp. 288-305, March 2007.

[3] M. Steiner and J. A. Smith, "Use of three-dimensional reflectivity structure for automated detection and removal of nonprecipitating echoes in radar data," J. Atmos Ocean Tech, vol. 19, no. 5, pp. 673-686, May 2002.

[4] N. Baraket and A. P. Bradley, "Rule extraction from SVMs: A review," Neurocomputing, vol. 74, no. 1, pp. 178-190, December 2010.
[5] C. Kessinger, S. Ellis, and J. Van Andel, "The radar echo classifier: A fuzzy logic algorithm for the WSR-88D," presented at $3^{\text {rd }}$ Conference on Artificial Applications to the Environmental Science, February 2003.

[6] Y. H. Kim, S. Kim, H. Y. Han, B. H. Heo, and C. H. You, "Real-time detection and filtering of chaff clutter from single-polarization doppler radar data," J. Atmos Ocean Tech, vol. 30, no. 5, pp. 873-895, May 2013.

[7] B. Schölkopf and A. J. Smola, "Learning with kernels: Support vector machines, regularization, optimization, and beyond," MIT press, 2002.

[8] C. J. Burges, "A tutorial on support vector machines for pattern recognition," Data Min Knowl Disc, vol. 2, no. 2, pp. 121-167, June 1998.

[9] C. F. Lin and S. D. Wang, "Fuzzy support vector machines," IEEE Trans. on Neural Networks, vol. 13, no. 2, pp. 464-471, March 2002.

[10] N. H. Barakat and A. P. Bradley, "Rule extraction from support vector machines: A sequential covering approach," IEEE Trans. Knowl Data En, vol. 19, no. 6, pp. 1729-741, June 2007.

[11] H. Núñez, C. Angulo, and A. Català, "Rule-based learning systems for support vector machines," Neural Process Lett, vol. 24, no. 1, pp. 1-18, August 2006.

[12] N. Barakat and J. Diederich, "Learning-based Rule-extraction from support vector machines," presented at The $14^{\text {th }}$ International Conference on Computer Theory and applications, September 2004.

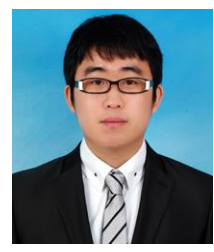

Hansoo Lee received his B.S. and M.S. degrees in electrical and computer engineering from Pusan National University, Busan, Korea, in 2010 and 2013, respectively. He is currently pursuing the Ph.D. degree at Pusan National University. His research interests include intelligent control, pattern recognition, classification, clustering and remote sensing.

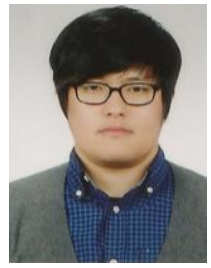

Yeongsang Jeong received the B.S. degree in information and communication engineering from Kyungnam University, Masan, Korea, in 2010. He received the M.S. degree in advanced engineering from Kyungnam University, Masan, Korea, in 2012. He is currently pursuing the Ph.D. degree at Pusan National University. His research interests include digital signal processing, fuzzy, recognition, adaptive noise control, soft computing.

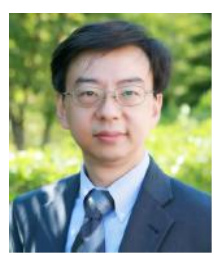

Sungshin Kim received his B.S. and M.S. degrees in Electrical Engineering from Yonsei University, Korea, in 1984 and 1986, respectively, and his Ph.D. degree in Electrical Engineering from the Georgia Institute of Technology, USA, in 1996. He is currently a professor at the Electrical Engineering Department, Pusan National University. His research interests include fuzzy logic controls, neuro fuzzy systems, neural networks, robotics, and intelligent systems. 